\title{
RECHTSSPRACHLICHE KOLLOKATIONEN IM DEUTSCH- POLNISCHEN VERGLEICH UND DEREN STELLENWERT IM AUSLANDSGERMANISTIKSTUDIUM
}

\section{STELLENWERT VON KOLLOKATIONEN IM AUSLANDSGERMANISTIKSTUDIUM}

Seit dem programmatischen Aufsatz von Franz Josef Hausmann mit dem Titel „Wortschatzlernen ist Kollokationslernen“ sind fast zwei Jahrzehnte vergangen. Es handelte sich dabei um ein Plädoyer für das syntagmatische Wortschatzlernen, das im Verhältnis zur Beschäftigung mit paradigmatischen Strukturen vernachlässigt werde (Hausmann 1984: 406). Sicherlich kann man beobachten, dass seit dieser Zeit das Bewusstsein für die Relevanz kollokativer Verbindungen beim Fremdsprachenerwerb gestiegen ist und es lässt sich mit Steyer feststellen, dass „Kollokationen auch im deutschsprachigen Raum aus ihrem Schattendasein an der Peripherie der Phraseologie herausgeholt und ihre Relevanz als Entitäten zwischen Sprachsystem und Sprachgebrauch, vor allem für den Fremdsprachenerwerb, ins allgemeine Bewusstsein gerückt" wurden (Steyer 2004: 92). Davon zeugen nicht nur zahlreiche theoretische Arbeiten zu Kollokationen aus fremdsprachendidaktischer Sicht (vgl. Hausmann 1984; Hartenstein 1996; Ludewig 2005; Reder 2006; Reder 2011), sondern auch bestimmte Instrumentarien, die zur Unterstützung des fremdsprachlichen Lernens in Form multilingualer Konkordanzprogramme entwickelt werden. Trotz alledem wird die sprachdidaktische Umsetzung der Erkenntnisse der Kollokationsforschung weiterhin mehrfach als unbefriedigend gewertet (vgl. Reder 2011: 131; Ludewig 2005: 22): Trotz des prominenten Stellenwerts von Kollokationen (vgl. Burger 2003: 52) werden sie nämlich in ,den Lehrmitteln und im Schulunterricht [...] kaum behandelt" (Häcki Buhofer 2011: 505).

Dabei stellen Kollokationen bereits in der Gemeinsprache eine Herausforderung für fremdsprachige Lerner dar. Sie ,sind als Marker für einen unauffällig formellen Sprachgebrauch ein zentraler Faktor der als normal, natürlich und richtig empfundenen Sprech- und Schreibweise in einer Sprache“ (Häcki Buhofer 2010: 65). Nach Hausmann sind Kollokationen insbesondere wegen ihrer Menge problemstiftend:

Wieviele Kollokationen muß ein Fremdsprachler aktiv beherrschen? Wenn man sich klar macht, dass eine große Zahl banalster Alltagsaussagen

Die Adresse des Autors: Instytut Filologii Germańskiej, Uniwersytet Opolski, Plac Staszica 1, 45-052 Opole, Polen. E-Mail: ksiezyk@uni.opole.pl 
kollokativ geregelt ist (Sagt man tun oder machen oder beides? [...]) und dass man nicht nur die kontrastiv abweichenden Kollokatoren lernen muss [...], sondern wegen der dadurch erfolgten Destabilisierung des Lerners auch die übereinstimmenden, dann wird klar, dass es sich um Zehntausende von Einheiten handelt (Hausmann 2003: 85).

Somit kann man den Eindruck gewinnen, die Sprache bestünde „fast mehr aus Formulierungen als auch Wörtern" (Hausmann 2007: 226).

Da die Fremdsprachenlerner eher Wortbeobachter als Kollokationensammler sind (vgl. Reder 2011: 138), sollten gängige Lehrwerke das Bewusstsein für die Kollokativität der Sprache schärfen, umso mehr, weil die „Kenntnis der Kollokationen einer Sprache [...] ein unverzichtbarer Baustein für ein unmarkiertes Sprechen und Schreiben [ist]“" (Häcki Buhofer 2011: 523).

Einer verbreiteten Auffassung zufolge seien Kollokationen im Gegensatz zu Idiomen bei der Rezeption unproblematisch, wie z.B. das unten stehende Zitat zeigt:

Kollokationen sind durchsichtig, aber nicht vorhersehbar. Ihre Idiomatizität fällt erst bei der Enkodierung auf. Die Idiomatizität der Redewendungen fällt bereits bei der Dekodierung auf (Hausmann 1995: 24; siehe auch Schwalm 1991: 17; Hausmann 2007: 218).

Demgegenüber wird in dem vorliegenden Beitrag mit Cedillo der Standpunkt vertreten, dass Kollokationsschwierigkeiten im fachsprachlichen Bereich sowohl bei der Hin-, als auch bei der Herübersetzung erwachsen können (vgl. Cedillo 2004: 33f.). Dies bestätigen die Ergebnisse studentischer Klausuren, bei denen polnische Studierende im sprachpraktisch angelegten Seminar zu Fachsprachenproblematik ebenso Probleme bei der Übersetzung ins Polnische haben. Sie führen etwa folgende fehlerhafte Verbindungen an, die im Polnischen nicht usuell sind: tworzyć testamenty anstatt sporzadzać testamenty ('Testamente errichten'), postawić wniosek anstatt złożyć wniosek ('Antrag stellen') oder narzucić karę anstatt nałożyć karę ('eine Strafe verhängen') (vgl. Księżyk 2012: 391). Auf ähnliche Fehler verweist Häcki Buhofer, dabei die Relevanz der richtigen Kombinierbarkeit herausstellend:

Gerade in domänengebundenen Sachbereichen [...], in denen sachadäquat und nicht allzu informell, sondern eher formell formuliert werden muss, sind die richtigen Kollokationen unverzichtbar [...] Die Busse $z u$ rücknehmen oder das Geld zurückgeben ist zwar verständlich, aber sehr laienhaft bzw. unprofessionell ausgedrückt (Häcki Buhofer 2010: 65; Herv. im Original).

Die Studierenden der Auslandsgermanistik selbst beteuern oftmals den Schwierigkeitsgrad fachsprachlicher Verbindungen, darauf verweisend, diese wären für sie 
auch in der Muttersprache erst zu erlernen. ${ }^{1}$ Die Wichtigkeit von Kollokationen gerade in Fachregistern betont u.a. Pieńkos, demzufolge den Leitern jeglicher Hochschulen und den Entscheidungsträgern bewusst gemacht werden sollte, dass sich die universitäre Ausbildung nicht allein auf den Erwerb der Allgemeinsprache beschränken könne (vgl. Pieńkos 1999: 200). Ähnlich wie Pieńkos positioniert auch PetkovaKessanlis die Probleme beim fremdsprachlichen Fachsprachenunterricht stärker in der Beherrschung von Kollokationen als der Terminologiebeherrschung:

[Es] zeichnet sich ab, dass die Defizite der Studierenden vermehrt im Bereich der fachsprachlichen Phraseologie zu verorten sind. Dabei erweist sich nicht die Beherrschung der fremdsprachlichen Terminologie als problematisch, sondern die notwendige Einbettung der Fachlexik in den fachsprachlichen Kontext. Denn diese Einbettung geschieht zum größten Teil mit Hilfe von sprachlichen Elementen aus der Gemeinsprache. Nun ist aber die Wahl dieser sprachlichen Mittel nicht willkürlich, sondern in vielerlei Hinsicht typisiert. Und eben diese fachsprachliche Ausdruckstypik bereitet Studierenden mit nicht-deutscher Muttersprache bei der Textproduktion große Schwierigkeiten (Petkova-Kessanlis 2010: 117).

\section{FACHSPRACHLICHE KOLLOKATIONEN IN AUSGEWÄHLTEN JURISTISCHEN LEHRBÜCHERN}

In dem vorliegenden Beitrag wird von dem engen, basisorientierten Kollokationsbegriff ausgegangen, wonach eine Kollokation aus einer determinierenden Basis und dem determinierten Kollokator besteht (vgl. Hausmann 2007: 218). Wie Häcki Buhofer bemerkt,

werden [Kollokationen] beim Sprechen bzw. Schreiben nicht ad hoc gebildet, sondern als zusammengehörige Syntagmen abgerufen. Das hat zur Folge, dass alternative, frei kombinierte Konstruktionen [...] als unpassend empfunden werden, obwohl sie semantisch und syntaktisch ebenso korrekt wären (Häcki Buhofer 2011: 506).

Die Tatsache, dass „ein Sachverhalt durch mehrere Wortkombinationen ausgedrückt werden kann, aber von den SprecherInnen/SchreiberInnen häufig ein und dieselbe gewählt wird" (Häcki Buhofer 2011: 522) zieht eine starke Routiniertheit der Sprache nach sich. Besonders deutlich kommt die Routiniertheit der Rechtssprache im interlingualen Vergleich zum Vorschein. So führt etwa Płomińska (2010: 152) Beispiele für Mehrwortverbindungen an, bei denen eine syntagmatische Übersetzung (auch unter Zuhilfenahme einschlägiger Wörterbuchäquivalente) an dem zielsprachlichen Usus vorbeigeht:

1 Vgl. auch Häcki Buhofer (2011: 522), derzufolge die Unkenntnis üblicher Wortverbindungen ,auch in der Erstsprache [gilt], wenn es um professionelle bzw. professionalisierte Ausdrucksweisen geht". 


\begin{tabular}{|l|l|l|l|}
\hline 1$)$ & $\begin{array}{l}\text { Weitere Familiensachen sind nicht } \\
\text { anhängig. }\end{array}$ & $\rightarrow$ & $\begin{array}{l}* \text { Inne sprawy rodzinne nie są zawisłe } \\
\text { przed sądem. } \\
\text { Usuell: Inne sprawy rodzinne nie są } \\
\text { przedmiotem rozpoznania przez sąd/nie } \\
\text { toczą się przed sądem/nie zawisły przed } \\
\text { sądem. ('Weitere Familiensachen werden } \\
\text { vor Gericht nicht geführt.') }\end{array}$ \\
\hline 2) & Die Frist beginnt mit dem Tag ... & $\rightarrow$ & $\begin{array}{l}* \text { Termin liczony jest od dnia ... } \\
\text { Usuell: Termin biegnie począwszy od } \\
\text { dnia ... ('Die Frist läuft ab dem...') }\end{array}$ \\
\hline 3$)$ & $\begin{array}{l}\text { Die Lieferung erfolgt } \\
\text { versandkostenfrei. }\end{array}$ & $\rightarrow$ & $\begin{array}{l}* \ldots \text { następuje bez opłat ... } \\
\text { Usuell: nie pobiera się kosztów } \\
\text { wysyłkowych. ('Es werden keine } \\
\text { Versandkosten erhoben.') }\end{array}$ \\
\hline
\end{tabular}

Tabelle 1: Unüblichkeit wörtlicher Übersetzungen (Quelle: Płomińska 2010: 152 und 155)

Anders als bei gemeinsprachlichen Kollokationen, deren Präferiertheit von Konventionen der Sprachgemeinschaft abhängt, fällt bei juristischen Mehrwortausdrücken vielfach auf, dass sie zudem vom Gesetzgeber normativ festgelegt sind. Kjær spricht in diesem Zusammenhang von normbedingten Wortverbindungen der Rechtssprache bzw. juristischen Nominationsstereotypen (vgl. Kjær 1994: 322f.). Ihr zufolge seien es institutionalisierte Wortverbindungen, die zwar bestimmte Kommutations- und Transformationsmöglichkeiten aufweisen, jedoch ,durch eine relative lexikalische Stabilität bzw. Variabilität gekennzeichnet [sind], die nur in ihrer Abhängigkeit von durch Gesetz oder Konvention festgelegten fachlichen Situationstypen erfaßbar ist" (Kjær 1994: 323). Dabei seien verschiedene Normierungsgrade zu unterscheiden. Eine schwache Normierung liegt etwa bei Deshalb ist Klage geboten vor, wonach diese Wendung etwa gegenüber Deshalb ist Klage notwendig/erforderlich bevorzugt wird. Von einer starken Normierung ist hingegen z.B. bei Angabe von Verdachtsgraden in der Strafprozessordnung auszugehen. So ist es zur Verhaftung eines Verdächtigen im Strafprozess erforderlich, den Verdachtsgrad mit dringend zu bezeichnen, wohingegen ein hinreichender oder begründeter Verdacht keine Voraussetzung für die Anordnung der Untersuchungshaft bildet (vgl. Kjær 1994: 324f. und 340).

In dem vorliegenden Beitrag sollen deutsche und polnische rechtssprachliche Kollokationen kontrastiv untersucht werden. Der Analyse werden Paralleltexte zu ausgewählten Rechtszweigen zugrunde gelegt. Den Ausgangspunkt bildet dabei das deutsche Lehrbuch Einführung in das deutsche Recht und die deutsche Rechtssprache (Simon/ Funk-Baker 2009), als Paralleltext dazu wird das polnische Lehrbuch Elementy prawa (Kocot 2007) herangezogen. Beide Werke sind an angehende Jurastudierende und interessierte Laien gerichtet und zeichnen sich durch eine ähnliche Makrostruktur aus. Wie Kjær zu Recht bemerkt, handelt es sich bei Lehrbüchern lediglich um Rechtstexte im weiteren Sinne. Im Gegensatz zu Rechtstexten sensu stricto, wie Verträgen, Urteilen 
oder Gesetzestexten besitzen Lehrbücher „keine Rechtsgültigkeit, mit ihnen werden keine Rechtshandlungen durchgeführt“ (Kjær 1995: 52). Dennoch kann angenommen werden, dass auch darin bestimmte usuelle und vom Gesetzgeber normativ festgelegte Verbindungen vorkommen. Ausgehend von den Kapiteln Verträge und andere Rechtsgeschäfte, Familien- und Erbrecht, Strafrecht und Verfahrensrecht wurden aus dem deutschen Text auf manuellem Wege 162 substantivisch-verbale kollokationsverdächtige Verbindungen extrahiert. ${ }^{2}$ Ausschlaggebend für die Extraktion waren das wiederholte Vorkommen bzw. die Halbkompositionalität der entsprechenden Wortkombinationen. Nach Entsprechungen im Polnischen wurde in dem polnischen Lehrbuch in den parallelen Kapiteln Podstawowe pojęcia prawne ('Juristische Grundbegriffe'), prawo cywilne ('Zivilrecht'), prawo rodzinne ('Familienrecht'), postepowanie cywilne ('Zivilverfahrensrecht'), und prawo karne ('Strafrecht') gesucht.

Die Analyse wurde auf substantivisch-verbale Verbindungen beschränkt, da sie als prototypisch bei Kollokationen gelten (vgl. Häcki Buhofer 2011: 508; ZenderowskaKorpus 2010: 164). Aus dem interlingualen Vergleich mit dem Polnischen wird deutlich, dass innerhalb der extrahierten Verbindungen nur 21 Kombinationen kompositionell übersetzbar sind, bei allen anderen (141 - d.h. 87\%) handelt es sich um kontextspezifische Übersetzungen der jeweiligen potentiellen Verbkollokatoren. Dieser Befund lässt den Eindruck entstehen, dass Kollokationen in den hier analysierten juristischen Lehrbüchern sehr häufig vorkommen. Daraus resultiert u.a. auch der Schwierigkeitsgrad, der solchen Verbindungen in der Fremdsprachendidaktik anlastet.

In der einschlägigen Literatur (vgl. Hausmann 2007; Cedillo 2004) wird das Kriterium der Halbkompositionalität, hier verstanden als nicht wörtliche Übertragung eines der Bestandteile von Mehrwortverbindungen, zum Teil als ausschlaggebend für die Einstufung als Kollokation angesehen, wie das folgende Zitat belegt:

Eine Schwierigkeit besteht jedoch bei der Vermittlung der Kollokationen darin, dass nicht nur prototypische Kollokationen existieren. So ist es nicht in jedem Einzelfall unumstritten, ob man der jeweiligen Wortverbindung den Kollokationsstatus anerkennen sollte. Bei Zweifelsfällen lässt sich jedoch eine kontrastive Betrachtungsweise heranziehen. Wortverbindungen, deren Bestandteile nicht wörtliche Äquivalente in der L1 haben, werden auf jeden Fall als Kollokationen betrachtet (Reder 2011: 137).

Zieht man neben der Nicht- bzw. Teilidiomatizität weitere Kriterien heran, die Kollokationen zugrunde gelegt werden (Polylexikalität, Stabilität, Assoziativität und Konventionalität), dann zeigt sich, dass aus der nicht-kompositionellen Übersetzung von Mehrwortverbindungen nicht automatisch auf deren Kollokativität zu schließen ist. Häcki Buhofer zufolge ist es nämlich nur hochwahrscheinlich, dass eine Kollokation vorliegt, wenn eine Übersetzung in eine andere bekannte Sprache nicht Wort für Wort

2 Die genannten Rechtsgebiete bildeten auch die thematische Grundlage für das sprachpraktisch angelegte Seminar Fachsprachen, das an der Universität Opole/Oppeln im akademischen Jahr 2011/2012 angeboten wurde und auf dessen Ergebnisse sich dieser Beitrag stützt. 
erfolgen kann (vgl. Häcki Buhofer 2011: 509). Daraus resultiert die Notwendigkeit, kollokationsverdächtige Verbindungen zusammen mit anderen analog aufgebauten Ausdrücken zu analysieren (vgl. Irsula Peña 1994: 33). Irsula Peña zufolge ist bei einer Kollokationsbasis nicht nur die Beziehung zu dem aktuellen Kollokator, sondern auch „die Beziehungen der Basis zu allen anderen potentiellen Kollokatoren als auch die Beziehungen der Kollokatoren zueinander“ (Irsula Peña 1994: 33) zu berücksichtigen. Entscheidend bei der Prüfung des Kollokationsstatus ist nämlich auch die Größe des Kombinationsbereichs der potentiellen Basen und Kollokatoren:

Je weniger Kollokatoren ein Basiswort hat, desto höher die Wahrscheinlichkeit von Phraseologizität - dies gilt auch für den fachsprachlichen Bereich (Worbs 1998: 112).

Bei der Analyse der extrahierten Verbindungen zeigt sich, dass Verbindungen, deren Bestandteile einen engen Kombinationsbereich aufweisen und daher aufgrund der Typikalität als kollokativ zu werten wären, eher eine Randerscheinung darstellen, etwa:

4) Akten (zum Verfahren) beiziehen - 'załączenie akt/dołączenie akt'. Bei dem Kollokator beiziehen ergibt die Suchanfrage im Digitalen Wörterbuch der Deutschen Sprache (DWDS), dass das Verb nur mit Akten in dem morphologisch-syntaktischen Strukturtyp Verb + Substantiv(Objekt) eine Verbindung eingeht.

5) von der Erbfolge ausgeschlossen werden/sein - 'być/zostać wyłączonym od dziedziczenia'. Bei dieser Verbindung zeigt sich wiederum, dass sich Erbfolge in der Funktion eines Präpositionalobjekts lediglich mit ausschließen verbindet, ferner kann man bei dieser Verbindung von einer Monosemierung ausgehen, da damit das Rechtsinstitut der Enterbung versprachlicht wird.

Viel häufiger handelt es sich bei den extrahierten Verbindungen um Kombinationen mit Verben, bei denen eine polyseme Bedeutungsvariante aktiviert wird, und deren Kollokativität erst vor dem Hintergrund ihrer Typikalität und Präferiertheit festgestellt werden kann. So wird etwa bei eine Straftat ausführen die Bedeutung 'verwirklichen' aktiviert, da die Kombination jedoch im DWDS überhaupt nicht nachweisbar ist und auch in der Suchmaschine Google keine hohe Trefferzahl liefert, kann sie als frei gewertet werden, zumal die Kombinationsbereiche der Bestandteile dieser Verbindung weit sind. Ähnlich ist auch das Verb ausschließen in seiner kontextuell spezifischen Bedeutung 'ausnehmen', 'nicht mit einbeziehen' hochproduktiv, sodass man hier laut Ludewig von freien Wortverbindungen sprechen könnte (vgl. Ludewig 2005: 89 und 91f.). Da innerhalb der extrahierten Verbindungen Gewährleistung und Haftung ausschließen der Frequenz nach typisch erscheinen, wobei Gewährleistung als Basis einen kleinen Kombinationsbereich aufweist und Haftung in Kombination mit ausschließen terminologisiert ist, ${ }^{3}$ kann man sie dennoch als Kollokationen einstufen. Im Gegensatz dazu kann

3 Vgl. http://www.rechtslexikon.net/d/haftungsausschluss/haftungsausschluss.htm; Stand: 4. 05. 2013. 
den Anspruch ausschließen als frei angesehen werden, da der Kombinationsbereich des Substantivs groß ist und ausschließen keine typische Verbindung damit eingeht.

Nicht als Kollokationen sind auch diejenigen Verbindungen zu werten, bei denen Verben zum Einsatz kommen, die typisch für die Amtssprache sind, die jedoch keine typischen restringierten Kombinationen darstellen, etwa: die gesetzliche Erbfolge tritt ein (im Sinne von 'erfolgen') oder die Scheidung erfolgt.

Innerhalb der extrahierten Verbindungen können insbesondere diejenigen Kombinationen als typischere Vertreter der Kategorie Kollokation gelten, bei denen der Kollokator im Zusammenhang mit dem Substantiv eine semantische Umdeutung erfahren hat und in dieser Bedeutung eine eingeschränkte Produktivität aufweist, etwa:

6) die Rechtskraft durchbrechen - 'pozbawić mocy prawnej/uchylić moc prawną'. Der Kollokator durchbrechen weist hier eine übertragene Lesart auf im Sinne von 'entziehen, aufheben'. Auch handelt es sich bei der Rechstkraftdurchbrechung um eine terminologisierte Verbindung. Sie tritt dann ein, „wenn über den Gegenstand eines rechtskräftigen Urteils erneut entschieden wird“. ${ }^{4}$

7) die Ehe scheitert - 'małżeństwo rozpada się'. Ähnlich kann auch in diesem Falle davon ausgegangen werden, dass diese Verbindung terminologisiert ist. Im BGB findet man sogar eine Legaldefinition dessen, wann eine Ehe gescheitert ist:

Eine Ehe kann geschieden werden, wenn sie gescheitert ist. Die Ehe ist gescheitert, wenn die Lebensgemeinschaft der Ehegatten nicht mehr besteht und nicht erwartet werden kann, dass die Ehegatten sie wiederherstellen (§ 1565 I BGB).

8) den Fall neu aufrollen - 'przekazać sprawę do ponownego rozpoznania'. Auch bei diesem Verbkollokator liegt eine semantische Spezifität vor, es wird übertragen im Sinne 'einer erneuten Entscheidung über einen Fall' verstanden.

Als Kollokationen sind ferner auch diejenigen Kombinationen einzustufen, bei denen ein Verb zwar mit mehreren Substantiven in Verbindung tritt, diese jedoch durch eine semantisch-begriffliche Verwandtschaft verbunden sind, so etwa bei eine Willenserklärung oder die Vaterschaft anfechten. Die Rechtshandlung der Anfechtung ist bei mangelhaften Willenserklärungen zulässig. Sie führt dazu, dass deren „Rechtsverbindlichkeit von Anfang an erlischt". ${ }^{5}$ Somit dürfte nicht nur die Verbindung eine Willenserklärung anfechten, sondern auch die Vaterschaft anfechten für kollokativ erachtet werden: zum einen wird die Vaterschaft bei nichtehelichen Kindern durch Anerkennung in Form einer Erklärung festgestellt und zum anderen weist das Substantiv einen engen Kombinationsbereich auf.

Vor diesem Hintergrund zeigt sich, dass innerhalb der 162 extrahierten Verbindungen lediglich 93 bei Berücksichtigung aller hier genannten Kriterien als Kollokationen gewertet werden könnten.

4 Quelle: http://www.lexexakt.de/glossar/rechtskraftdurchbrechung.php; Stand: 18. 05. 2013.

5 http://www.rechtslexikon.net/d/anfechtung/anfechtung.htm; Stand 23. 04. 2013. 


\section{VERMITTLUNG FACHSPRACHLICHER KOLLOKATIONEN}

Da bei substantivisch-verbalen Kollokationen den Verben die Funktion eines Kollokators zukommt und dieser ,nicht ohne die Basis definiert, gelernt und übersetzt werden [kann - F.K.]“ (Hausmann 2007: 218), gebührt den Verben in der Fremdsprachendidaktik besondere Beachtung. Innerhalb der extrahierten Kombinationen fällt auf, dass es sich dabei kaum um rechtsspezifische Verben handelt, überwiegend kommen darin Kollokatoren vor, die auch in der Gemeinsprache vorkommen, im Vergleich dazu jedoch eine semantische Weiterentwicklung erfahren haben. Dem Vorschlag von Zenderowska-Korpus folgend, kann es gerade in diesen Fällen eine effektive Lehr- und Lernstrategie sein, „von solchen Kollokationen auszugehen, die in der Gemeinsprache und in den Fachsprachen vorkommen" (Zenderowska-Korpus 2010: 170). Im textbezogenen Lernen kommt es daher darauf an, den Studierenden die rechtssprachlichen Kollokationen bewusst zu machen, indem sie die semantische Spezifizität der Verbkollokatoren im Verhältnis zu der Gemeinsprache - wie die folgende Gegenüberstellung exemplifiziert - ergründen.

\begin{tabular}{|c|c|}
\hline Gemeinsprache & Rechtssprache \\
\hline $\begin{array}{l}\text { den Schlüssel abgeben ('übergeben/ } \\
\text { aushändigen') }\end{array}$ & $\begin{array}{l}\text { eine Willenserklärung abgeben } \\
\text { ('einreichen') }\end{array}$ \\
\hline die Tür abschließen ('versperren') & $\begin{array}{l}\text { einen Vertrag abschließen ('sich } \\
\text { [vertraglich] binden') }\end{array}$ \\
\hline eine Freundin anrufen ('telefonieren') & das Gericht anrufen ('vor Gericht gehen') \\
\hline $\begin{array}{l}\text { seine Gründe auseinandersetzen } \\
\text { ('darlegen') }\end{array}$ & $\begin{array}{l}\text { (gemeinschaftliches) Vermögen } \\
\text { auseinandersetzen ('aufteilen') }\end{array}$ \\
\hline ein Kind einholen ('erreichen') & ein Gutachten einholen ('sich geben lassen') \\
\hline das Buch einstellen ('stellen, einordnen') & $\begin{array}{l}\text { das Verfahren einstellen ('unterbrechen, } \\
\text { beendigen') }\end{array}$ \\
\hline den Arm erheben ('hoch heben') & $\begin{array}{l}\text { Anklage erheben ('vorbringen, geltend } \\
\text { machen') }\end{array}$ \\
\hline $\begin{array}{l}\text { das Fahrgeld erstatten ('ersetzen, } \\
\text { zurückzahlen') }\end{array}$ & eine Anzeige erstatten ('einreichen') \\
\hline ein Kind führen ('geleiten') & einen Namen führen ('tragen, haben') \\
\hline ein Fenster verhängen ('verdecken') & Geldstrafen verhängen ('anordnen') \\
\hline
\end{tabular}

Tabelle 2: Semantische Weiterentwicklung rechtssprachlicher Kollokatoren

Eine weitere empfehlenswerte Lernstrategie könnte auf der Sensibilisierung für die reihenbildende Wirkung der rechtssprachlichen Kollokatoren beruhen. Ähnlich wie eine Willenserklärung abgegeben wird, werden auch einzelne Typen von Erklärungen 
abgegeben, etwa eine Sorgeerklärung. Analog wie die Berufung eingelegt wird, werden auch andere Rechtsmittel, wie Revision oder Einspruch eingelegt. Ansprüche, eine Anklage oder Klage werden wiederum erhoben. Kann eine Ehe scheitern, so ist diese Verbindung auch bei einer Lebenspartnerschaft möglich, die mit der Rechtsform der Ehe gewisse Parallelen in den dadurch entfalteten Rechtswirkungen zeigt (vgl. Simon/ Funk-Baker 2009: 92).

Da sich die Präferiertheit kollokativer Verbindungen ,im Sprachvergleich am ehesten plausibel machen [lässt]“ (Lüger 2004: 49) und es „keine intensivere Auseinandersetzung mit einem Text [gibt,] als wenn man ihn übersetzen muss" (Nussbaumer 2007: 40), empfiehlt es sich ferner, Kollokationen im fremdsprachigen Text zu erkennen, sie zu übersetzen und sie sprachproduktiv einzusetzen. Wie Ludewig betont, unterstützen:

kontrastive Gegenüberstellungen den Betrachter nicht nur hinsichtlich der (zu erlernenden) Fremdsprache [...], sondern [sensibilisieren] ihn darüber hinaus auch in Hinblick auf die eigene, bereits verinnerlichte Muttersprache [...]. Der Sprachvergleich schärft den Blick des Betrachters also nicht nur für die Kollokativität der Fremdsprache, sondern insbesondere auch für die Kollokativität seiner Muttersprache und fördert so das Bewusstsein von der eigenen Muttersprache (Ludewig 2005: 118).

In diesem Rahmen lassen sich beispielsweise zweisprachige Übungen einsetzen, bei denen für polnische Kollokationen deutsche Entsprechungen zu nennen sind oder umgekehrt, etwa:

\begin{tabular}{|ll|l|}
\hline 1. & einen Erben einsetzen & a. $\quad$ pobierać podatki \\
\hline 2. & $\begin{array}{l}\text { ein Sachverständigengutachten } \\
\text { einholen }\end{array}$ & b. ustanowić spadkobiercę \\
\hline 3. & Steuern erheben & c. $\quad$ złożyć doniesienie \\
\hline 4. & Leistungen erbringen & d. $\quad$ zasięgnąć opinii biegłego \\
\hline 5. & Anzeige erstatten & e. $\quad$ spełniać świadczenia \\
\hline
\end{tabular}

Tabelle 3: Zweisprachige Zuordnungsübung

Dabei gilt es zu betonen, dass es nicht darauf ankommt, isolierte, zusammenhanglose Kollokationen zu übersetzen. Vielmehr sollen die Studierenden aufgefordert werden, bei der Arbeit mit Texten zu juristischer Problematik kollokative Verbindungen ausfindig zu machen und auf der Grundlage paralleler Texte Äquivalente finden zu lassen. Trotz bestehender Nachteile des Übersetzens im Fremdsprachenunterricht erscheint die Übersetzungsmethode bei der Kollokationsvermittlung insbesondere wegen der bereits angesprochenen semantischen Umdeutung der Verbkollokatoren als geeignet (vgl. Ludewig 2005: 119). 


\section{FAZIT}

Die aus den analysierten Lehrwerken extrahierten Wortkombinationen erweisen sich aus kontrastiver Sicht überwiegend als halbkompositionell, indem sie eine andere Bedeutung als in der Gemeinsprache aktivieren und die verbalen Bestandteile zumeist nur kontextspezifisch übertragbar sind. Die Folge ist, dass aus der fremdsprachendidaktischen Perspektive in der Rechtssprache zunächst fast alles semantisch intransparent vorkommen kann. Was vorerst arbiträr und idiosynkratisch scheint, erweist sich allerdings bei der Prüfung der Kombinationsbereiche der Bestandteile der extrahierten Verbindungen zum Teil als frei, da die Produktivität vieler Verben nicht restringiert ist und man nicht immer mit typischen oder normativ festgelegten Kombinationen zu tun hat. Eine Vertrautheit mit den fachsprachlichen Kollokationen erfordert somit ein Eintauchen in den fachsprachlichen Diskurs. Infolge eines Perspektivwechsels von der Gemein- zur Fachsprache können auch die Proportionen, was die Kollokativität bzw. Freiheit der fachsprachlichen Verbindungen anbelangt, etwas modifiziert werden.

\section{Literatur}

\section{Primärliteratur}

KOCOT, Wojciech (Hrsg.) (2007) Elementy prawa. Warszawa: Difin.

SIMON, Heike/Gisela FUNK-BAKER (2009) Einführung in das deutsche Recht und die deutsche Rechtssprache. 4. neubearbeitete Aufl. München u.a.: Beck.

\section{Sekundärliteratur}

BURGER, Harald (22003) Phraseologie. Eine Einführung am Beispiel des Deutschen. Berlin: Schmidt.

BGB = Bürgerliches Gesetzbuch mit Allgemeinem Gleichbehandlungsgesetz, Beurkundungs $G$, BGB-Informationspflichten-Verordnung, Einführungsgesetz, Erbbaurechtsgesetz, Lebenspartnerschaftsgesetz, Produkthaftungsgesetz, Unterlassungsklagengesetz und Wohnungseigentumsgesetz (2012). Textausgabe mit ausführlichem Sachverzeichnis und einer Einführung von Universitätsprofessor Dr. Helmut Köhler. 70. überarbeitete Auflage. München: dtv.

CEDILLO, Ana Caro (2004) Fachsprachliche Kollokationen. Ein übersetzungorientiertes Datenbankmodell Deutsch-Spanisch. Tübingen: Narr. (Forum für Fachsprachenforschung, 63).

ĎURČO, Peter (Hrsg.) (2010) Feste Wortverbindungen und Lexikographie. Kolloquium zur Lexikographie und Wörterbuchforschung. Berlin/New York: de Gruyter. (Lexicographica Series Maior, 138).

DUŚ, Magdalena/Grażyna ZENDEROWSKA-KORPUS (Hrsg.) (2010) Fachsprachenpropädeutik im Germanistikstudium. Częstochowa: Wydawnictwo Wyższej Szkoły Lingwistycznej.

HÄCKI BUHOFER, Annelies (2010), „Die lexikographische Erfassung von Kollokationen in Dialektwörterbüchern.“ In: P. Ďurčo (Hrsg.), 65-76. 
HÄCKI BUHOFER, Annelies (2011) „Lexikographie der Kollokationen zwischen Anforderungen der Theorie und der Praxis." In: S. Engelberg/A. Hollter/K. Proost (Hrsg.), Sprachliches Wissen zwischen Lexikon und Grammatik. Berlin/Boston: de Gruyter, 505-531.

HARTENSTEIN, Klaus (1996) „Faustregeln als Lernhilfen für Lexemkollokationen (vorgeführt am Beispiel des Deutschen, Englischen, Französischen und Russischen).“ In: id. (Hrsg.), Aktuelle Probleme des universitären Fremdsprachenunterrichts. Hamburg: Zentrales Fremdspracheninstitut. (ZFI-Arbeitsberichte, 11), $83-134$.

HAUSMANN, Franz Josef (1984) „Wortschatzlernen ist Kollokationslernen. Zum Lehren und Lernen französischer Wortverbindungen." Praxis des neusprachlichen Unterrichts 31/4, 395-406.

HAUSMANN, Franz Josef (1995) „Von der Unmöglichkeit der kontrastiven Lexikologie.“"In: H.-P. Kromann/A. L. Kjær (Hrsg.), 19-23.

HAUSMANN, Franz Josef (2003) „Kollokationen in der Fachsprache: Schwerpunkt Französisch." In: U. O. H. Jung/A. Kolesnikova (Hrsg.), Fachsprachen und Hochschule. Forschung-Didaktik-Methodik. Frankfurt am Main: Lang. (Bayreuther Beiträge zur Glottodidaktik, 9), 84-92.

HAUSMANN, Franz Josef (2007) „Die Kollokationen im Rahmen der Phraseologie - Systematische und historische Darstellung.“ Zeitschrift für Anglistik und Amerikanistik 55/3, 217-234.

IRSULA PEÑA, Jesús (1994) Substantiv-Verb-Kollokationen. Kontrastive Untersuchungen Deutsch-Spanisch. Frankfurt am Main u.a.: Lang.

KJÆR, Anne Lise (1994) „Zur kontrastiven Analyse von Nominationsstereotypen der Rechtssprache deutsch - dänisch.“ In: B. Sandig (Hrsg.), 317-348.

KJÆR, Anne Lise (1995) „Vergleich von Unvergleichbarem. Zur kontrastiven Analyse unbestimmter Rechtsbegriffe.“ In: H.-P. Kromann/A. L. Kjær (Hrsg.), EUROPHRAS 92. Tendenzen der Phraseologieforschung. Bochum: Brockmeyer, 39-56.

KROMANN, Hans-Peder/Anne Lise KJÆR (Hrsg.) (1995) Von der Allgegenwart der Lexikologie: kontrastive Lexikologie als Vorstufe zur zweisprachigen Lexikographie. Tübingen: Niemeyer. (Lexicographica: Series maior, 66).

KSIĘŻYK, Felicja (2012) „Zur Bedeutung fachsprachlicher Anteile im Auslandsgermanistikstudium." In: T. Tinnefeld et al. (Hrsg.), Hochschulischer Fremdsprachenunterricht. Anforderungen, Ausrichtung, Spezifik. Saarbrücken: htw saar, 383-397.

LUDEWIG, Petra (2005) Korpusbasiertes Kollokationslernen. Computer-Assisted Language Learning als prototypisches Anwendungsszenario der Computerlinguistik. Frankfurt am Main: Lang.

LÜGER, Heinz-Helmut (2004) „Kollokationen - zwischen Arbitrarität und Kompositionalität.“ In: I. Pohl/K.-P. Konerding (Hrsg.), 45-66.

NUSSBAUMER, Markus (2007) „Gesetzestext und Wissenstransfer? - Welche Funktionen Gesetzestexte erfüllen müssen und wie man sie optimieren kann.“ In: D. Heller/K. Ehlich (Hrsg.), Studien zur Rechtskommunikation. Bern u.a.: Lang. (Linguistic Insight. Studies in Language and Communication, 56), 18-45. 
PETKOVA-KESSANLIS, Mikaela (2010) „Fachsprachliche Kollokationen im linguistischen Diskurs.“ In: P. Durčo (Hrsg.), 115-126.

PIEŃKOS, Jerzy (1999) Podstawy juryslingwistyki. Język w prawie - Prawo $w$ języku. Warszawa: MUZA.

PŁOMIŃSKA, Małgorzata (2010) „Routineformeln in deutschen und polnischen juristischen Texten.“ In: M. Duś/G. Zenderowska-Korpus (Hrsg.), 149-161.

POHL, Inge/Klaus-Peter KONERDING (Hrsg.) (2004) Stabilität und Flexibiltät in der Semantik: Strukturelle, pragmatische und kognitive Perspektiven. Frankfurt am Main u.a.: Lang.

REDER, Anna (2006) Kollokationen in der Wortschatzarbeit. Wien: Praesens.

REDER, Anna (2011) „Kommen Kollokationen in Mode? Kollokationskonzepte und ihre mögliche Umsetzung in der Didaktik." Linguistik online 47, 3/2011, 131-140. http://www.linguistik-online.de/47_11/reder.pdf [4. Mai 2013].

SCHWALM, Gisela (1991) „Zum Problem der Kollokation in Gemein- und Fachsprache im Kontext der Übersetzung." TEXTconTEXT 6, 163-182.

STEYER, Kathrin (2004) „Kookkurrenz. Korpusmethodik, linguistisches Modell, lexikographische Perspektiven." In: id. (Hrsg.), Den Nagel auf den Kopf treffen. Wortverbindungen - mehr oder weniger fest. Berlin/New York: de Gruyter, 87-116.

WORBS, Erika (1998) „Was ist fachsprachliche Phraseologie? Überlegungen am Beispiel der deutschen und polnischen Börsensprache." In: H. Rothe/P. Thiergen (Hrsg.), Polen unter Nachbarn. Polonistische und komparatistische Beiträge zur Literatur und Sprache. Köln/Weimar/Wien: Böhlau, 99-127.

ZENDEROWSKA-KORPUS, Grażyna (2010) „Kollokationen in den Fachsprachen.“ In: M. Duś/G. Zenderowska-Korpus (Hrsg.), 163-176.

Rechtslexikon. 4. Mai 2013. http://www.rechtslexikon.net.

Lexexakt. 18. Mai 2013. http://www.lexexakt.de.

Digitales Wörterbuch der deutschen Sprache. 18. Mai 2013. www.dwds.de.

\section{Zusammenfassung \\ RECHTSSPRACHLICHE KOLLOKATIONEN IM DEUTSCH- POLNISCHEN VERGLEICH UND DEREN STELLENWERT IM AUSLANDSGERMANISTIKSTUDIUM}

Entgegen der verbreiteten Ansicht, dass Kollokationen insbesondere bei der Übersetzung in die Nicht-Muttersprache Probleme bereiten, ihre Rezeption dagegen ungehindert erfolgt, wird im vorliegenden Beitrag davon ausgegangen, dass dies bei Fachwendungen nicht der Fall ist. Das Phänomen der Kollokationen soll durch eine Analyse ausgewählter juristischer Lehrbücher näher beleuchtet werden. Angesichts der Schwierigkeiten, die fachsprachliche Kollokationen auch Muttersprachlern bereiten, 
werden einige Wege zum kompetenteren Umgang mit diesen sprachlichen Phänomenen vorgestellt.

Schlüsselbegriffe: Kollokationen, Deutsch als Fremdsprache, Fachsprachendidaktik, Rechtslinguistik, Wortschatzlernen.

\section{Povzetek}

NEMŠKO-POLJSKA PRIMERJAVA KOLOKACIJ PRAVNEGA JEZIKA IN NJIHOV POMEN PRI ŠTUDIJU NEMŠČINE KOT TUJEGA JEZIKA

Kljub uveljavljenemu mnenju, da kolokacije predstavljajo probleme predvsem pri prevajanju $\mathrm{v}$ nematerni jezik, njihova recepcija pa težav ne povzroča, avtorica tega članka izhaja iz predpostavke, da kaj takega za strokovne kolokacije ne moremo trditi. Fenomen kolokacij skuša na podlagi analize izbranih pravnih učbenikov nadrobneje osvetliti. Ker strokovne kolokacije povzročajo težave tudi rojenim govorcem, bo avtorica $\mathrm{v}$ prispevku predstavila nekaj načinov, kako se je tega jezikovnega fenomena mogoče lotiti kompetentneje.

Ključne besede: kolokacije, nemščina kot tuji jezik, didaktika strokovnega jezika, pravna lingvistika, učenje besedišča. 